\title{
Is a soft nuclear equation of state extracted from heavy-ion data incompatible with pulsar data?
}

\author{
Irina Sagert ${ }^{1}$, Mirjam Wietoska ${ }^{1}$, Jürgen Schaffner-Bielich ${ }^{1} \ddagger$, \\ Christian Sturm ${ }^{2}$ \\ ${ }^{1}$ Institut für Theoretische Physik/Astrophysik, J. W. Goethe Universität, \\ D-60438 Frankfurt am Main, Germany \\ ${ }^{2}$ Institut für Kernphysik, J. W. Goethe Universität, D-60438 Frankfurt am Main, \\ Germany \\ E-mail: schaffner@astro.uni-frankfurt.de
}

\begin{abstract}
We discuss the recent constraints on the nuclear equation of state from pulsar mass measurements and from subthreshold production of kaons in heavy-ion collisions. While recent pulsar data points towards a hard equation of state, the analysis of the heavy-ion data allows only for soft equations of state. We resolve the apparent contradiction by considering the different density regimes probed. We argue that future measurements of global properties of low-mass pulsars can serve as an excellent cross-check to heavy-ion data.
\end{abstract}

\section{Introduction}

There has been a tremendous activity recently with regard to the properties of dense nuclear matter in high-density astrophysics of compact stars and in heavy-ion physics. The maximum mass of neutron stars for example is intimately related to the underlying stiffness of the nuclear equation of state (EoS), see e.g. [1] and references therein for a recent review.

More than 1600 pulsars, rotation-powered neutron stars, are presently known. New timing measurements of binary pulsars point towards large masses and correspondingly to a nuclear EoS usually considered to be rather stiff. For the white dwarf-pulsar system $\mathrm{J} 0751+1807$ a mass of $M=(2.1 \pm 0.2) M_{\odot}(1 \sigma)$ and $M=(1.6-2.5) M_{\odot}(2 \sigma)$ was found by Nice et al. [2]. Özel derives a mass limit $M \geq(2.10 \pm 0.28) M_{\odot}$ from x-ray bursts of the neutron star EXO 0748-676 [3] stating that soft equations of state are ruled out.

On the other hand, particle production and collective effects in heavy-ion collisions are also considered to be a probe of the underlying nuclear EoS. The subthreshold production of kaons as measured by the KaoS collaboration ([4] and [5]) were investigated in transport models (see [6] and for a most recent reinvestigation see [7] as $\ddagger$ talk given at the international conference on 'Nuclear Physics in Astrophysics III', Dresden, March 26-31, 2007 
well as [8]) coming to the conclusions that the nuclear EoS should be considerably soft above normal nuclear matter density.

We investigate in detail this apparent contradiction by considering several families of nuclear equations of state, with regard to compression modulus and symmetry energy, and their impact on the properties of compact stars taking into account the recent data on kaon production in heavy ion collisions. We note that a combined analysis of other heavy-ion data (not on kaon production data) and compact stars has been performed in [9]. In particular, the role of the density region relevant for subthreshold production and the related Schrödinger equivalent potentials are delineated. In addition, the importance of hyperons in dense neutron star matter with regard to known hypernuclear properties are contrasted with pulsar mass measurements. Also, the influence of a possible quark phase on the mass-radius relation of compact stars is discussed, in particular in response to the recent analysis by Özel (see [10]).

\section{The nuclear equation of state and high-density astrophysics}

The nuclear EoS serves as a crucial input for modelling astrophysical systems with extreme matter densities as core-collapse supernovae (for a review see [11]), neutron star mergers (see e.g. [12, 13]), proto-neutron star evolution [14] and cold neutron stars [15]. The required density range is huge, spanning densities from about $10^{-10} n_{0}$ to up to $10 n_{0}$, where $n_{0}=0.15 \mathrm{fm}^{-3}$ stands for the normal nuclear matter density (the low-density region is of particular importance for dynamical systems, core-collapse supernovae and neutron star mergers). The temperature scale of interest spans from the keV range up to $50 \mathrm{MeV}$, for neutron star mergers even larger temperatures might be possible which depends sensitively on the stiffness of the nuclear EoS. The nuclear equations of state presently used in most dynamical simulations are the ones of Lattimer and Swesty [16] and Shen et al. [17]. On the other hand, there are dozens of nuclear equations of state used for describing proto-neutron stars and cold neutron stars, see e.g. [18] and references therein.

Stars with a zero-age main sequence mass of more than 8 solar masses end in a core-collapse supernova (type II). A new generation of simulation codes has been developed during the last few years including first steps towards a full 3D-treatment and Boltzmann neutrino transport. These improved models of stellar core collapse have found no explosions initially and the question on the missing physic ingredient was raised (see [19]). We quote from this reference: "... the models do not explode. This suggests missing physics, possibly with respect to the nuclear equation of state ...". (After the conference the situations has changed substantially. In particular, the newest simulation found indeed that the models do explode. A 15 solar mass progenitor star finally exploded after about $600 \mathrm{~ms}$ due to the standing accretion shock instability, dubbed SASI, see [20].)

The remnants of core-collapse supernovae (type II and type Ib/Ic) are neutron stars,

compact massive objects with typical masses of $(1-2) M_{\odot}$ and radii of about $10 \mathrm{~km}$. 
The link between pulsars, rotation-powered neutron stars, and supernova remnants is most evident for the crab pulsar and the crab nebula, the supernova remnant of the historical supernova of 1054 A.D. Nowadays, more than 1600 pulsars are known, the number is increasingly steadily due to recent detailed radio pulsar scans. However, the list of well determined pulsar masses is restricted to a few dozens, to binary systems (for a most recent compilation from radio observations see [21]). The best determined masses from relativistic double neutron star systems are between $1.44 M_{\odot}$ for B1913+16 (the Hulse-Taylor pulsar) and (1.18 \pm 0.02$) M_{\odot}$ for the pulsar J1756-2251 [22].

Of enormous interest for the nuclear EoS is the report on the measurement of massive neutron stars in pulsar-white dwarf systems, in particular for the pulsar J0751+1807 the mass constraints $M=(2.1 \pm 0.2) M_{\odot}(1 \sigma)$ and $M \geq 1.6 M_{\odot}(2 \sigma)$ have been reported [2]. The larger the mass pulsar limit the more can the nuclear EoS be constrained at high densities. Also measurements of neutron star radii will help to learn more about the physics of the interior of neutron stars. The isolated neutron star RXJ 1856, the closest known neutron star, emits a rather perfect x-ray black-body spectrum. However, combined with the optical flux measurements by the Hubble Space Telescope, a two temperature fit is needed. A small soft temperature implies a rather large radius so that the optical flux is right giving a conservative lower limit for the radiation radius of $R_{\infty}=16.5 \mathrm{~km}(\mathrm{~d} / 117 \mathrm{pc})[23]$. Note, that such a large radiation radius rules out most of the nuclear equations of state presently discussed in the literature, not only for quark matter but also for ordinary nuclear matter composed of neutrons, protons and leptons only.

The X-Ray burster EXO 0748-676 has been recently reanalysed by Özel [3] who concludes that the mass and radius are constrained by $M \geq(2.10 \pm 0.28) M_{\odot}$ and $R \geq(13.8 \pm 1.8) \mathrm{km}$. One important ingredient is the redshift extracted from spectral lines [24]. Both, mass and radius, are quite large so that very soft nuclear equations of state, could be ruled out. The appearance of new degrees of freedom in nuclear matter, as hyperons or kaon condensates, can considerably soften the nuclear EoS as the new degrees of freedom fill new low-lying Fermi levels. However, quark matter is a completely new phase with entirely new properties, so that the quark matter EoS can be rather stiff. Hence, pure quark stars as well as hybrid stars, neutron stars with nuclear and quark matter components, can be well within the above mass and radius limits [10].

In any case, there seems to be growing evidence from pulsar data that the nuclear EoS should be hard at high densities.

\section{The nuclear equation of state and heavy-ion physics}

On the other hand, kaon production at subthreshold energies in heavy-ion collisions arrives at the conclusion that the nuclear EoS is rather soft (see also the talks by Peter Senger and Christian Fuchs [5, 6])!

Nucleus-nucleus collisions at relativistic energies offer the unique possibility to study experimentally the properties of dense nuclear matter in the laboratory. In a reaction 
between two heavy nuclei at beam energies around $1 \mathrm{AGeV}$ nuclear matter will be compressed up to three times saturation density. Therefore, heavy-ion experiments provide the possibility to obtain information on the nuclear EoS at high baryon densities.

In order to obtain information on the high-density phase of a nucleus-nucleus collision it is necessary to measure particles which are created predominantly in this phase. At the energy domain of the SIS at GSI Darmstadt $K^{+}$mesons seem to be promising candidates due to their production mechanism and their long mean free path in nuclear matter. The propagation of $K^{+}$mesons in nuclear matter is characterised by the absence of absorption (as they contain an antistrange quark) and hence they are almost undisturbed messengers.

The production of $K^{+}$mesons requires multiple nucleon-nucleon collisions or secondary collisions such as $\pi N \rightarrow K^{+} \Lambda$ at subthreshold beam energies ( $E_{\text {beam }}=$ $1.58 \mathrm{GeV}$ for $\left.N N \rightarrow K^{+} \Lambda N\right)$. Since these multi-step processes occur preferentially at high baryon densities $K^{+}$mesons are expected to be produced mostly during the high density phase of the reaction. Hence, the $K^{+}$yield depends sensitively on the maximum baryon densities reached within the reactions which is determined by the stiffness of the nuclear EoS. Therefore, $K^{+}$mesons are expected to be well suited to obtain information on the nuclear EoS at high baryon densities.

Early transport calculations predicted that the $K^{+}$yield from $\mathrm{Au}+\mathrm{Au}$ collisions at subthreshold energies will be enhanced by a factor of about two if a soft rather than a hard EoS is assumed [25]. More recent calculations (IQMD [26], RQMD [6]) were performed with two values for the compression modulus of $200 \mathrm{MeV}$ and $380 \mathrm{MeV}$ which correspond to a "soft" or "hard" nuclear EoS, respectively. The transport models take into account a repulsive $K^{+} N$ potential and use momentum-dependent Skyrme forces to determine the compressional energy per nucleon (i.e. the energy stored in compression) as a function of the baryon density.

The assumed repulsive $K^{+} N$ potential depends nearly linear on the baryonic density [27] and thus reduces the $K^{+}$yield accordingly. On the other hand, at subthreshold beam energies the $K^{+}$mesons are created in secondary collisions involving two or more particles and hence the production of $K^{+}$mesons depends at least quadratically on the density. To disentangle these two competing effects the KaoS Collaboration [28] has studied $K^{+}$production in a very light $\left({ }^{12} \mathrm{C}+{ }^{12} \mathrm{C}\right)$ and a heavy collision system $\left({ }^{197} \mathrm{Au}+{ }^{197} \mathrm{Au}\right)$ at different beam energies near threshold. Due to pile-up of nucleons the maximum baryonic density in $\mathrm{Au}+\mathrm{Au}$ reactions is significantly higher than in the light collision system $\mathrm{C}+\mathrm{C}$. Moreover, the maximum baryonic density reached in $\mathrm{Au}+\mathrm{Au}$ reactions depends on the compression modulus of nuclear matter whereas in $\mathrm{C}+\mathrm{C}$ collisions this dependence is very weak [6]. Additionally, the use of the $\mathrm{Au} / \mathrm{C}$ ratio provides the advantage that uncertainties of the experiment (beam normalisation etc.) as well as of the transport model calculations (elementary cross sections etc.) cancel partly.

The ratio of the $\mathrm{K}^{+}$production excitation functions for $\mathrm{Au}+\mathrm{Au}$ and $\mathrm{C}+\mathrm{C}$ reactions obtained by the KaoS Collaboration increases with decreasing beam energy [4]. The 
comparison with transport model calculations demonstrates clearly that only the calculations based on a soft nuclear EoS reproduce the trend of the experimental data [6].

\section{Probing the nuclear equation of state}

In this section, we examine the constraints from astrophysical and heavy-ion data using an empirical nucleon-nucleon interaction. As ansatz for the energy per particle we take the standard Skyrme-type parameterisation, which is also used in the above mentioned analysis of the heavy-ion data:

$$
\epsilon / n=m_{N}+E_{0}^{k i n}+\frac{A}{2} \cdot u+\frac{B}{\sigma+1} u^{\sigma}+S_{0} \cdot u \cdot\left(\frac{n_{n}-n_{p}}{n}\right)^{2}
$$

where $u=n / n_{0}$. The parameters $A, B, \sigma$ are fixed by the nuclear matter properties, i.e. by normal nuclear matter density $n_{0}$, the binding energy $E / A$, the compression modulus $K$, and the asymmetry term by the asymmetry energy $S_{0}$ at $n_{0}$. The pressure is determined by the thermodynamic relation $P=n^{2} d(\epsilon / n) / d n$, which fixes the EoS underlying the transport model calculations of [6, 7]. We note that the equation of state can become acausal for $\sigma>2$. With this EoS at hand, we can check whether or not low compressibilities are ruled out by neutron star mass measurements or not.

Figure 1 shows the results for the maximum mass configuration of neutron stars in dependence of the compression modulus $K$. Plotted is the maximum mass, at certain points the corresponding radius, maximum speed of sound, and the maximum density are denoted in addition. The maximum central density reached is $n_{c} \approx 5 n_{0}$ for $K=200$ $\mathrm{MeV}$. There is a small variation of the radius on the compression modulus $K$ and the EoS stays causal, i.e. $c_{s}^{2} \leq 1$, up to $K=350 \mathrm{MeV}$. We also find that there is only a slight dependence on the symmetry energy $S_{0}$, up to $\Delta M= \pm 0.05 M_{\odot}$ for low values of the compression modulus for $S_{0}=(28-32) \mathrm{MeV}$. Most importantly, however, the maximum mass can be larger than two solar masses, $M \geq 2 M_{\odot}$, for a a compression modulus above $K \approx 190 \mathrm{MeV}$. Hence, even 'soft' equations of state in terms of a small compression modulus can give large neutron star masses!

The above analysis has been repeated using relativistic mean-field (RMF) models. It is important to realize that the essential input to transport models is the nonrelativistic Schrödinger equivalent potential, which determines the propagation of the nucleons in the transport simulation, not the EoS directly (this is not important for the non-relativistic model used above). We derive a non-relativistic nucleon potential from the Skyrme parameterisation corresponding to $K=200 \mathrm{MeV}$. We compare this nucleon potential to that of RMF parameterisations including a possible vector selfinteraction term and allow for only those parameter sets which have a Schrödinger equivalent potential compatible with the one from the Skyrme parameterisation. We find indeed RMF parameter sets fulfilling that constraint which have maximum neutron star masses around two solar masses if only nucleons and leptons are taken into account. Hence, soft (non-relativistic) potentials can be also compatible with pulsar masses! A 


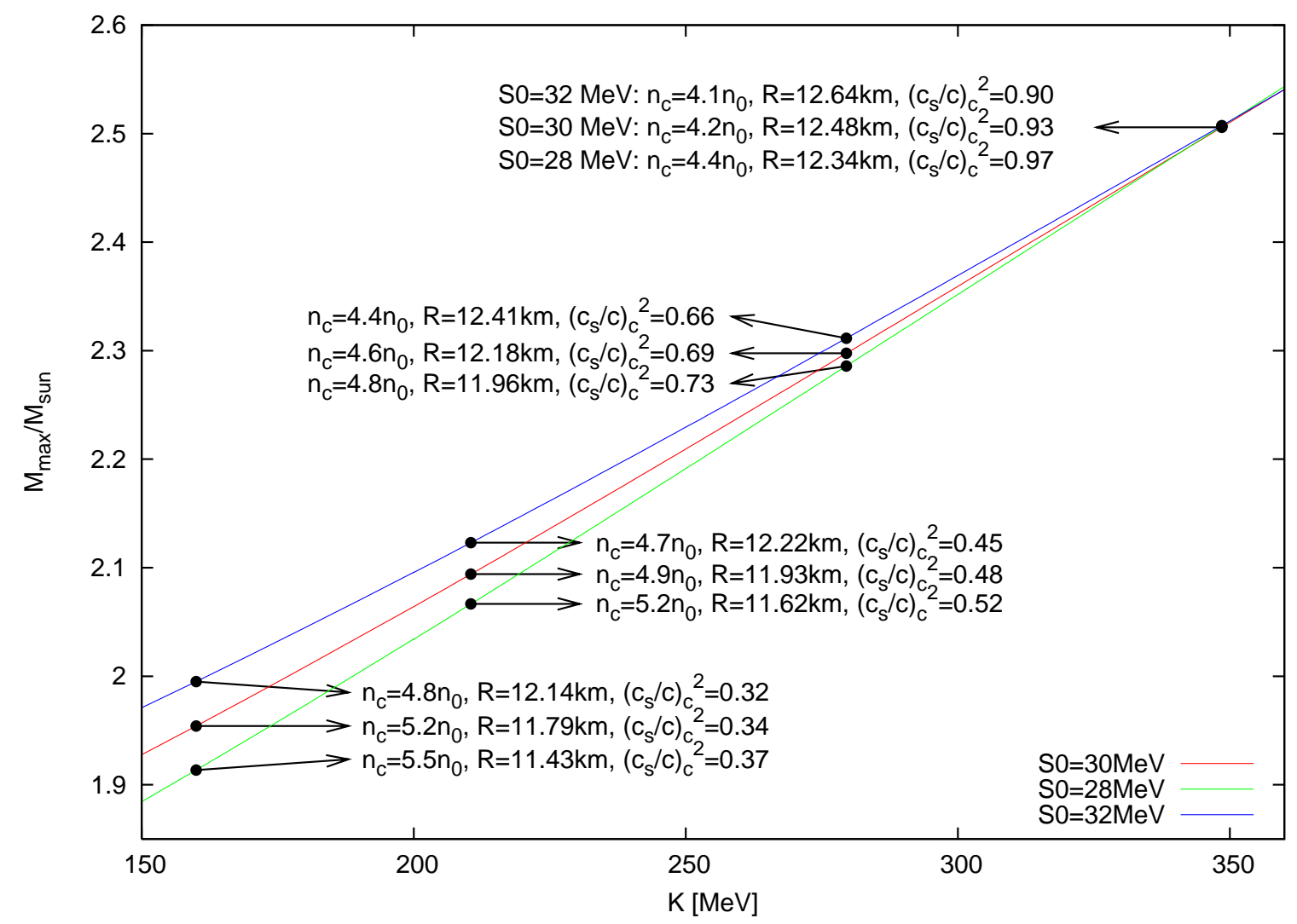

Figure 1. The maximum mass as a function of the compression modulus $K$ for nucleons and electrons only in $\beta$-equilibrium, choosing the symmetry energy to be $S_{0}=28,30$, and $32 \mathrm{MeV}$. For some points on the curve the central density, the radius, and the maximum speed of sound squared are denoted. A compression modulus of $K \geq 190 \mathrm{MeV}$ is sufficient to arrive at a maximum mass above $2 M_{\odot}$.

most recent analysis using relativistic Brueckner-Hartree-Fock models arrives at a similar conclusion, see the contribution by C. Fuchs [8].

However, the maximum density reached is about $5 n_{0}$. At such large densities, various models describing nuclei as well as hypernuclear data find consistently that hyperons are present already at $n=2 n_{0}$ in neutron star matter, see e.g. the discussion in [29]. Also, kaon condensates as well as quark matter could be present in the core of neutron stars at such large densities. Hyperons drastically reduces the maximum mass possible (we refer to [30] for a particular nice plot demonstrating this effect). Hyperons are a new degree of freedom, therefore the overall reduced pressure does not allow for large neutron star masses. Indeed, we find that the inclusion of hyperons reduces the maximum masses to about $1.75 M_{\odot}$ and below in RMF models. For the RMF parameter sets with sufficiently soft Schrödinger equivalent potentials compatible with the KaoS data masses below $1.6 M_{\odot}$ (the lower limit from J0751+1807) are found, although masses above $1.44 M_{\odot}$ are possible. It is remarkable that the presence of hyperons have such an effect on the maximum mass, although they are only present above $2 n_{0}$. 
Hence, we conclude that the relevant density region probed by the maximum mass and mass limits from pulsar data is most likely above $2 n_{0}$. So the high density EoS is crucial for the maximum mass which is controlled by unknown physics (hyperons, kaon condensation, quark matter). Kaon production data from heavy-ion experiments on the other hand are sensitive to moderate densities of around $2 n_{0}$ only. Different density regions are probed so that present heavy-ion data and pulsar mass limits can not be compared directly. However, this finding immediately points towards a real comparison: to check for the global properties of low-mass neutron stars with moderate maximum densities! Within the empirical nucleon-nucleon interaction it turns out that a $1.2 M_{\odot}$ neutron star probes at maximum the EoS up to $n \approx 2 n_{0}$, the right maximum density so that exotic states are likely to be not present. The lowest measured mass of a pulsar is the one of J1756-2251 with $(1.18 \pm 0.02) M_{\odot}[22]$. A measurement of the radius of such a low-mass neutron star would allow for a more consistent cross-check between the nuclear EoS derived from heavy-ion experiments and pulsar observations.

\section{Summary}

We have demonstrated that a soft nuclear EoS as extracted from kaon production data is not in contradiction with recent pulsar data. The EoS above $n \approx 2 n_{0}$ determines the maximum mass of neutron stars which is controlled by unknown high-density physics (hyperons, kaon condensation, quarks). Neutron stars with hyperons have rather low maximum masses, so that he presence of hyperons makes it substantially more difficult to comply with heavy-ion and pulsar data. Properties of low-mass neutron stars $\left(M \approx 1.2 M_{\odot}\right)$, on the other hand, are entirely controlled by the EoS up to only $n \approx 2 n_{0}$. Measurements of the radius of low-mass pulsars open therefore the exciting opportunity for a consistent cross-check between heavy-ion and pulsar data on the properties of the nuclear equation of state at moderately high densities.

\section{Acknowledgments}

This work is supported in part by the Gesellschaft für Schwerionenforschung mbH, Darmstadt, Germany. Irina Sagert gratefully acknowledges support from the Frankfurt Institute for Advanced Studies and the Helmholtz Research School for Quark Matter Studies.

\section{References}

[1] J. M. Lattimer and M. Prakash, Phys. Rept. 442, 109 (2007), astro-ph/0612440.

[2] D. J. Nice, E. M. Splaver, I. H. Stairs, O. Löhmer, A. Jessner, M. Kramer, and J. M. Cordes, Astrophys. J. 634, 1242 (2005), astro-ph/0508050.

[3] F. Özel, Nature 441, 1115 (2006), astro-ph/0605106.

[4] C. Sturm et al. (KaoS), Phys. Rev. Lett. 86, 39 (2001), nucl-ex/0011001.

[5] talk given by Peter Senger, see the contribution to these proceedings. 
[6] C. Fuchs, A. Faessler, E. Zabrodin, and Y.-M. Zheng, Phys. Rev. Lett. 86, 1974 (2001), nuclth/0011102.

[7] C. Hartnack, H. Oeschler, and J. Aichelin, Phys. Rev. Lett. 96, 012302 (2006), nucl-th/0506087.

[8] C. Fuchs, arXiv:0706.0130 [nucl-th] (2007), contribution to these proceedings.

[9] T. Klähn, D. Blaschke, S. Typel, E. van Dalen, A. Faessler, C. Fuchs, T. Gaitanos, H. Grigorian, A. Ho, E. Kolomeitsev, et al., Phys. Rev. C 74, 035802 (2006), nucl-th/0602038.

[10] M. Alford, D. Blaschke, A. Drago, T. Klähn, G. Pagliara, and J. Schaffner-Bielich, Nature 445, E7 (2006), astro-ph/0606524.

[11] H.-T. Janka, K. Langanke, A. Marek, G. Martínez-Pinedo, and B. Müller, Phys. Rept. 442, 38 (2007), astro-ph/0612072.

[12] S. Rosswog and M. B. Davies, Mon. Not. Roy. Astron. Soc. 345, 1077 (2003), astro-ph/0110180.

[13] R. Oechslin, H.-T. Janka, and A. Marek, Astron. Astrophys. 467, 395 (2007), astro-ph/0611047.

[14] J. A. Pons, S. Reddy, M. Prakash, J. M. Lattimer, and J. A. Miralles, Astrophys. J. 513, 780 (1999), astro-ph/9807040.

[15] F. Weber, Prog. Part. Nucl. Phys. 54, 193 (2005), astro-ph/0407155.

[16] J. M. Lattimer and F. D. Swesty, Nucl. Phys. A535, 331 (1991).

[17] H. Shen, H. Toki, K. Oyamatsu, and K. Sumiyoshi, Nucl. Phys. A637, 435 (1998), nuclth/9805035.

[18] F. Weber, R. Negreiros, and P. Rosenfield, arXiv:0705.2708 [astro-ph] (2007).

[19] R. Buras, M. Rampp, H. T. Janka, and K. Kifonidis, Phys. Rev. Lett. 90, 241101 (2003), astro$\mathrm{ph} / 0303171$.

[20] H.-T. Janka, A. Marek, and F.-S. Kitaura, arXiv:0706.3056 [astro-ph] (2007).

[21] I. H. Stairs, J. Phys. G 32, S259 (2006).

[22] A. J. Faulkner, M. Kramer, A. G. Lyne, R. N. Manchester, M. A. McLaughlin, I. H. Stairs, G. Hobbs, A. Possenti, D. R. Lorimer, N. D’Amico, et al., Astrophys. J. 618, L119 (2005), astro-ph/0411796.

[23] J. E. Trümper, V. Burwitz, F. Haberl, and V. E. Zavlin, Nucl. Phys. Proc. Suppl. 132, 560 (2004), astro-ph/0312600.

[24] J. Cottam, F. Paerels, and M. Mendez, Nature 420, 51 (2002), astro-ph/0211126.

[25] J. Aichelin and C. M. Ko, Phys. Rev. Lett. 55, 2661 (1985).

[26] G. Hartnack, L. Sehn, J. Jaenicke, H. Stöcker, and J. Aichelin, Nucl. Phys. A580, 643 (1994).

[27] J. Schaffner-Bielich, I. N. Mishustin, and J. Bondorf, Nucl. Phys. A625, 325 (1997).

[28] P. Senger et al. (KaoS), Nucl. Instrum. Meth. A327, 393 (1993).

[29] J. Schaffner-Bielich, M. Hanauske, H. Stöcker, and W. Greiner, Phys. Rev. Lett. 89, 171101 (2002), astro-ph/0005490.

[30] N. K. Glendenning and S. A. Moszkowski, Phys. Rev. Lett. 67, 2414 (1991). 